\title{
Data Handling Techniques in Online Semiconductor Technology CAD Laboratory
}

\author{
http://dx.doi.org/10.3991/ijoe.v7iS2.1753 \\ Ananda Maiti and B. K. Tripathy \\ Vellore Institute of Technology, Vellore TN, India
}

\begin{abstract}
Modern semiconductor manufacturing is recognized as a specialized field in electrical engineering curricula. Teaching micro- and nanoelectronics in university environment is a challenging task and a new framework is needed. An integrated measurement-based microelectronics laboratory along with technology computer aided design (TCAD) simulation laboratory has been developed and is in use for imparting hands-on laboratory experience to the students. An internet-based laboratory management system for monitoring and control of a real-time measurement system interfaced via a dedicated local computer is discussed. TCAD process/device simulations generate a vast amount of data and handling of the data is a challenge for web-based applications. A simple architecture for implementation, visualization and analyses of the generated data from process/device simulations is reported.
\end{abstract}

Index Terms-Microelectronics education, online laboratory, TCAD, data management

\section{INTRODUCTION}

Technology-enhanced learning is becoming important in higher education worldwide. In particular, enhancing engineering education is an emerging field of research as it involves various disciplines which aim to resolve the pedagogical issues of laboratory education. With the availability of broadband Internet, currently, many research groups are exploring various ways to support and augment the distance laboratory learning activities. Important outcomes of these efforts are the online laboratories. In traditional laboratories most of the equipment is only used during the laboratory session and remain idle for most of the time of the year. Online laboratories are ideal for sharing the laboratory resources. Online laboratories use interactive experiments which are available over the Internet [1]. These laboratories are commonly divided into two groups; simulation and remote measurement-based laboratories in which real hardware equipment are used and controlled remotely.

Computer Aided Design (CAD) of circuits is highly complicated and demands a lot of computing resources. To improve the competence of electronics, education of CAD requires support of numerous software tools. As an example, CADENCE tool, demand high performance computers, a lot of disc memory, and the maintenance of the software. Very few Universities can afford such laboratories. However, current capabilities of the Internet open the ways of using any CAD system via any computer connected to the Internet. Recent research on integrated circuits design environments shows that the World Wide Web (WWW) is a potential media to support the design environment. Distance CAD laboratory which uses softwares distributed by the EUROPRACTICE has been reported [2]. The students in microelectronics courses perform their laboratory exercises here. The CAD design course is essential for basic microelectronic education as it results in a deeper understanding of VLSI CAD.

With the advancements in semiconductor manufacturing; it is becoming increasingly difficult for the VLSI designers to optimize the design without considering the effects of the VLSI processes (process variation). Technology CAD (TCAD) is used in the semiconductor industry via two main approaches. The first approach is in predictive simulation, which is used in a conceptual device design phase for the prediction of trends in process options, device operation and circuit behavior [3].

The second approach is to use experimental data to calibrate the models in the simulator and then to use the calibrated tools in the optimization phase of the development process. TCAD tools are available at different levels for different applications. The different tools play different roles in any technology development strategy. TCAD also comprises many other elements, including equipment simulation, pattern transfer simulation and compact modelling. Simulation of the fabrication steps of microelectronic devices and integrated circuits is a technique that is used extensively in silicon IC manufacturing to speed product development and reduce the development costs. Semiconductor technology relies essentially on technology CAD support during process development and process optimization. Visualization is one of the biggest advantages of TCAD tools. During the process simulation the evolution of actual cross-sections of the structure can be seen [4]. The conventional TCAD laboratory involves mostly process/device simulation for both undergraduate and graduate students. Development of a state-of-the-art laboratory module on TCAD with tutorials is necessary.

The proposed simulation-based TCAD laboratory has the following objectives; the use of TCAD tools allows students to learn the fundamentals of device processing in a virtual environment including the determination of SPICE parameters; the ability to develop skills on semiconductor process design experience (very similar to the actual experience obtained from a fabrication laboratory); and the ability to acquire proficiency with the use of TCAD tools and issues relating to analog and digital circuit design. Two major components of TCAD are: (a) Process simulation and (b) Device simulation. Process simulation is modeling of semiconductor manufacturing processes. Process simulation allows one to investigate the possible process steps prior to actual fabrication. Process simulation includes basic unit steps such as oxidation, 
implantation, diffusion, etching, growth, and deposition [5].

\section{A. Process Simulation: SUPREM}

SUPREM is an advanced 2D process simulator originally developed for submicron silicon structures. It provides cross-sections of arbitrary device structures based on physical models for ion implantation, diffusion, and oxidation and annealing. It is designed to interface with other programs that accurately simulate etching and deposition of thin films on the semiconductor surface, although it includes basic models for these processes as well. Process simulator SUPREM generates both printed and graphical outputs that describe the simulation results.

\section{B. Device Simulation: BIPOLE}

BIPOLE is a quasi-two-dimensional device simulator, developed at the University of Waterloo, Canada, which specifically focuses on rapid prediction of terminal electrical characteristics of bipolar transistors. Programme input comprises fabrication data such as mask dimensions, impurity profiles and physical parameters such as carrier lifetime. The calculation is based on the variable boundary regional approach using one-dimensional transport equations. Two-dimensional and quasi-cylindrical edge effects are handled by combining the vertical one-dimensional analysis with a coupled one-dimensional horizontal analysis of the transport equations in the base region. It is then used to solve the transport equations for diffusion and drift in the presence of arbitrary recombination in quasi-neutral regions. This scheme is extremely fast in terms of computing time. BIPOLE can also be used for accurate avalanche multiplication and breakdown studies in high-speed transistors with shallow collector regions and heterojunction structures, including graded base Ge fraction in SiGe devices, and emission across the thin interfacial oxide layer in a poly-Si emitter and offers a facility for automatic parameter extraction for SPICE models.

\section{Virtual Wafer Fabrication (VWF)}

Virtual Wafer Fabrication (VWF) is used for performing Design of Experiments (DOE) and Optimization Experiments. Split-lots can be used in various pre-defined analysis methods. Split parameters can be defined for any process, device, and parasitic extraction and circuit simulators. All simulations are carried out in parallel either on a cluster of workstations. Much attention has been given to creation of user-friendly pre- and post-processors, data storage and retrieval, and other components that are needed for an online laboratory management system.

TCAD process/device simulations generate a vast amount of data and handling of the data is a challenge for web-based applications. This is particularly true when bandwidth of the network plays a very crucial role. In this work, we focus on the emerging field of scientific visualization, which is proving to be very helpful in analyzing the generated data from process/device simulations, as well as recent developments in the geometrical modelling of 3D structures, which is needed for characterizing complicated device structures and analyzing the data. The design and development of a suitable data base to handle the generated data is described. The integration of measurement-based microelectronics and VLSI engineering laboratory with simulation-based technology CAD laboratory is described. The management system allows the remote students to conduct remote experiments, perform monitoring and control of the experimental setup and collect data from the experiment through the network link. The data can be imported in the simulation-based TCAD laboratory for SPICE parameter extraction. System Architecture of TCAD Laboratory is shown in Figure 1.

\section{THE LABORATORY}

Major steps for remote online laboratory development are: (a) the design of the experiment, (b) remote control and operation of the instruments or softwares (for example, via LabVIEW), (c) conversion to web applications and (d) launching the experiments on the internet. A modular online remote laboratory typically consists of several hardware-based experiments which need to be made available always to students. The laboratory (available at http://lod.iitkgp.ernet.in/netlab/) is an initiative to develop an online measurement-based Microelectronics and VLSI Engineering Laboratory integrated with simulation-based Technology CAD laboratory (see Figures 2 and 3).

As the internet is accessible 24 hours a day, it has a very high potential for Computer Aided Instruction (CAI). Java is a platform independent language, which ensures that all the included applications are able to run on any kind of system found on the Internet. As an objectoriented programming language, Java allows the reuse of source code. This feature is very important for the course, since one of its sections deals with CAD tools construction.

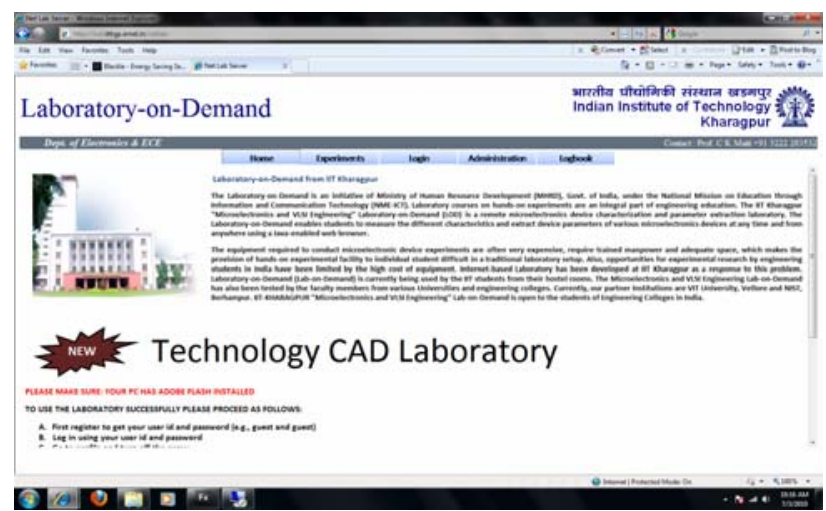

Figure 1. Integrated TCAD Laboratory home page.

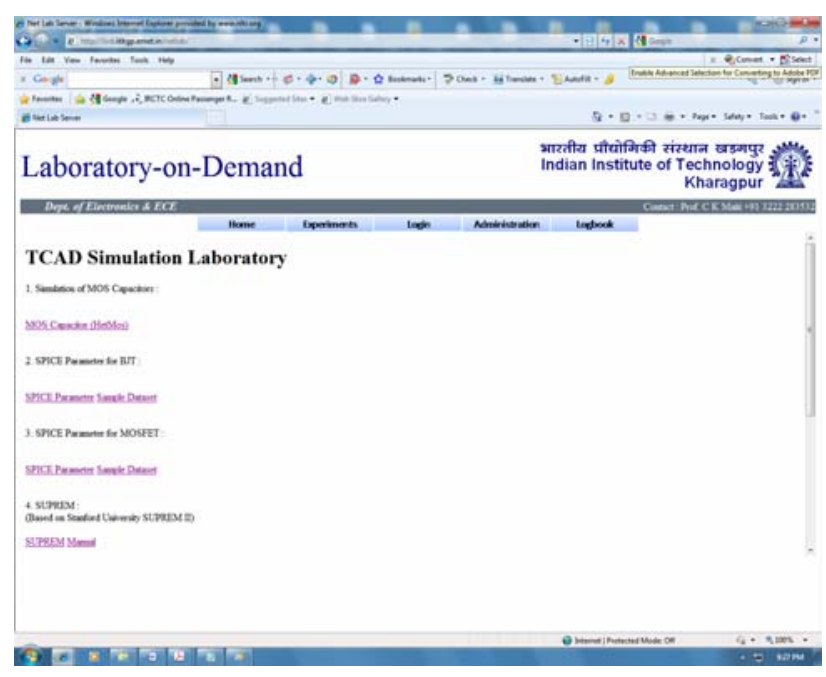

Figure 2. Available experiments in the TCAD Lab. 


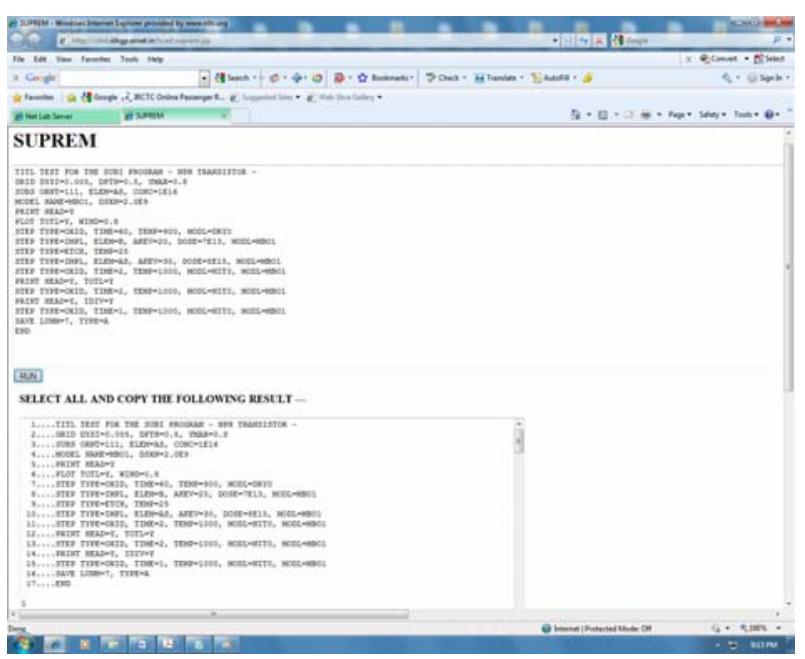

Figure 3. TCAD Laboratory SUPREM II web page.

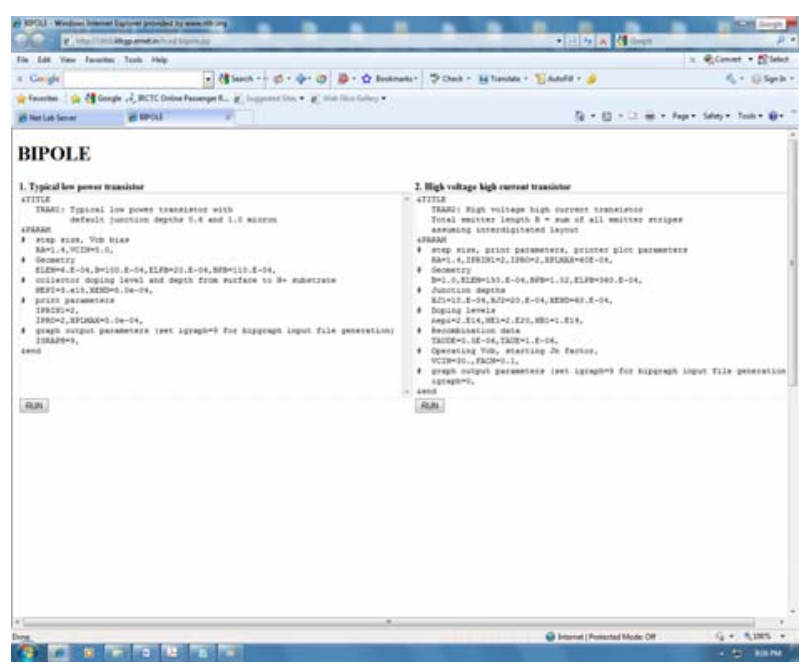

Figure 4. TCAD Laboratory BIPOLE web page.
Hypertext Markup Language was chosen to provide an atmosphere that eases access to the information resources and that allows interaction by the student. The Adobe Flex program running on the student's PC sends a request for connection to the program running on the Web Server. The program then sends the connection request to the controller as well as accepts the request from the student. Online TCAD laboratory gives the student, even at the undergraduate level, a chance to learn about realistic silicon wafer processing via hands-on simulation experiments.

\section{DATA MANAGEMENT}

Now let us see the architecture of the schedulers to handle requests to the TCAD Laboratory (Figure 1). The server side program has three parts: Main scheduler, Temporary File or work Area and Stored programs as discussed in Section 1.

\section{A. Main Scheduler}

The main scheduler accepts requests from the client and parses them. Then it decides which program to run. After deciding the program to run, it creates a process exclusively for the request.

\section{B. Request Processes}

The process then proceeds with the following steps :

1. Create a temporary copy of the program in the temporary work area

2. Write down all inputs in a text file or in the specific input format of the program

3. Then it runs the program to create an output file.

The output of the program may or may not be in the $\mathrm{XML}$ format. If it is in the xml format then it is directly sent back to the user client where it is displayed using a web browser. If the output is not in the XML format for e.g. in text format then file is opened and parsed for im-

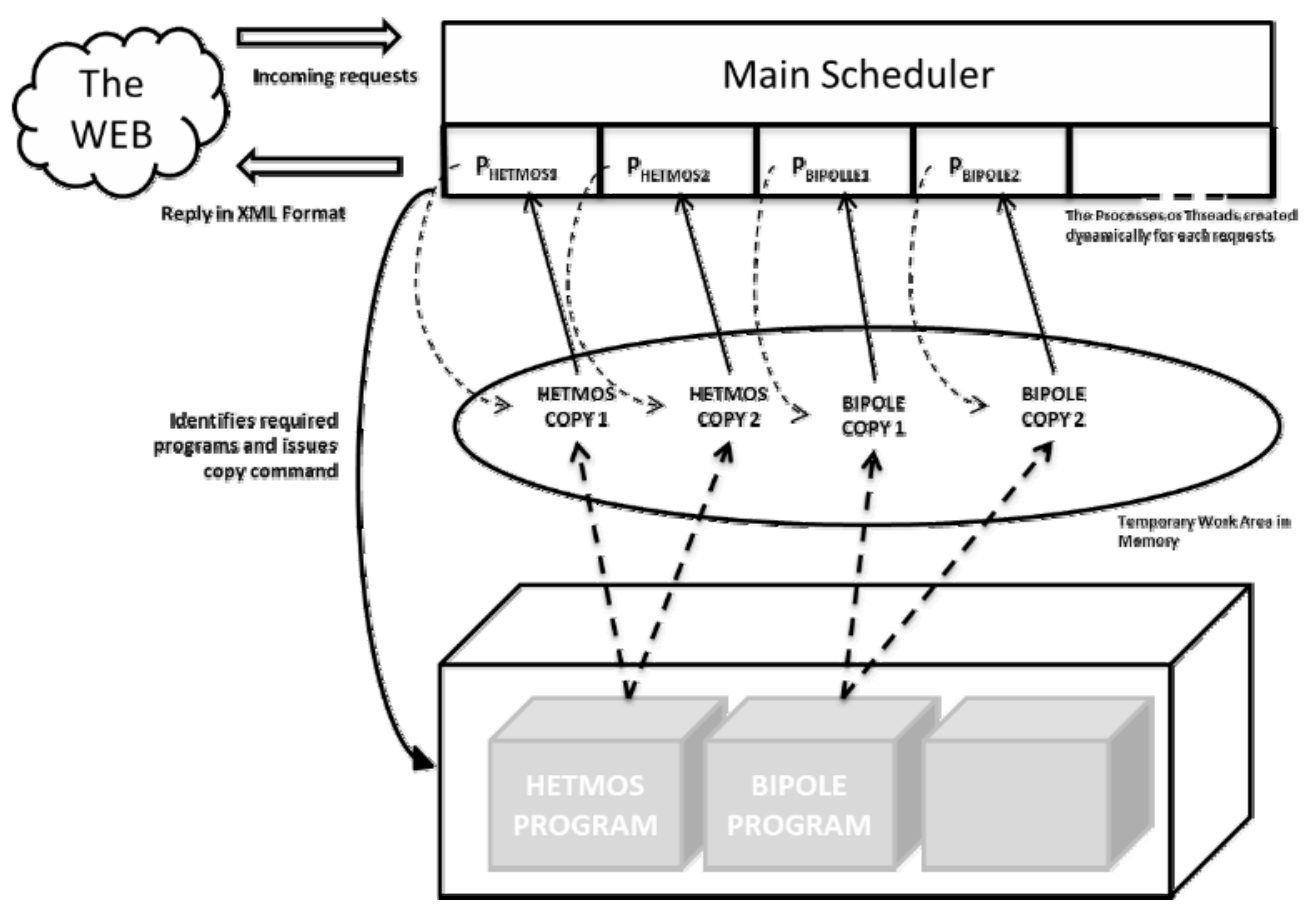

Figure 5. System Architecture of TCAD Laboratory. 
portant information that are required to be send to the user. Upon completion of parsing, the output is send back to the user client.

Since all the programs run independently, they can be run simultaneously without any problem of deadlock or starvation.

The request from a client can be composed of

- A set of parameters for simulations.

- A data set from an experiment for parameter extraction

In the first case the parameters are sent from users to client and the user client is generally in HTML formats (see Figure 3 and 4). This gives the user a natural environment to write their parameters in conventional terms and regulations. However this can be elaborately designed using the Adobe Flex. In the second case the user performs some experiment from the MVL (Microelectronics and VLSI) lab. This helps display the data in a graphical manner along with the parameters and the extracted features.

The data that is generated from the MVL experiment generate is an array of data which is represented as a table (see figure 6). The data can be copied and pasted in CSV (comma separated values) format in text files for further processing. The data can also be pasted directly to the TCAD application front end. The front end then parses the data to generate a tabular value depending upon the experiment and as suited for processing in the back end processing (see figure 7). Once the data has been parsed it is send to the back end server which runs the main scheduler as discussed earlier. This forms the basis of the Integrated TCAD Laboratory as discussed in the next section.

\section{Integrated TCAD Laboratory}

In the following, an integrated measurement-based the Microelectronics and VLSI Engineering Laboratory with simulation-based Technology CAD (TCAD) laboratory is described. Measurements are fundamental to an understanding of any semiconductor device. To teach device design and developing concepts the Microelectronics and VLSI Engineering laboratory has been developed. We use tools (LabVIEW) from the National Instruments (NI) electronics education platform to teach microelectronic devices concepts. The device characterization module makes use of two distinct pieces of laboratory hardware/software. The heart of the system is the LCR meter and a semiconductor device parameter analyzer (Agilent E4980 and Agilent 4156C). Broadly speaking, the units generate the user-specified inputs to a connected test device, perform the test and collect the measured data. The bipolar device characterization module which makes use of a single instrument (Agilent 4156C) for the characterization of bipolar junction transistors (BJT) involving five experiments sequentially. The Experiment Module for BJT characterization consists of the following experiments:

- Gummel Plot

- Static Collector Characteristics

- Emitter Resistance

- Collector Resistance

- Current gain vs. Collector Current

The complete range of semiconductor DC parameters can be quickly and accurately evaluated with 4156C stand-alone instrument. One of the most important steps in evaluating bipolar transistor parameters is measuring collector current and base current as a function of baseemitter voltage (Gummel plot). The measured Gummel characteristics of an NPN transistor are shown in Figure 6. The success of a VLSI circuit design depends on the device models used to describe the device behavior. It is thus imperative that these devices are accurately characterized so that the most accurate model parameter set for the de-

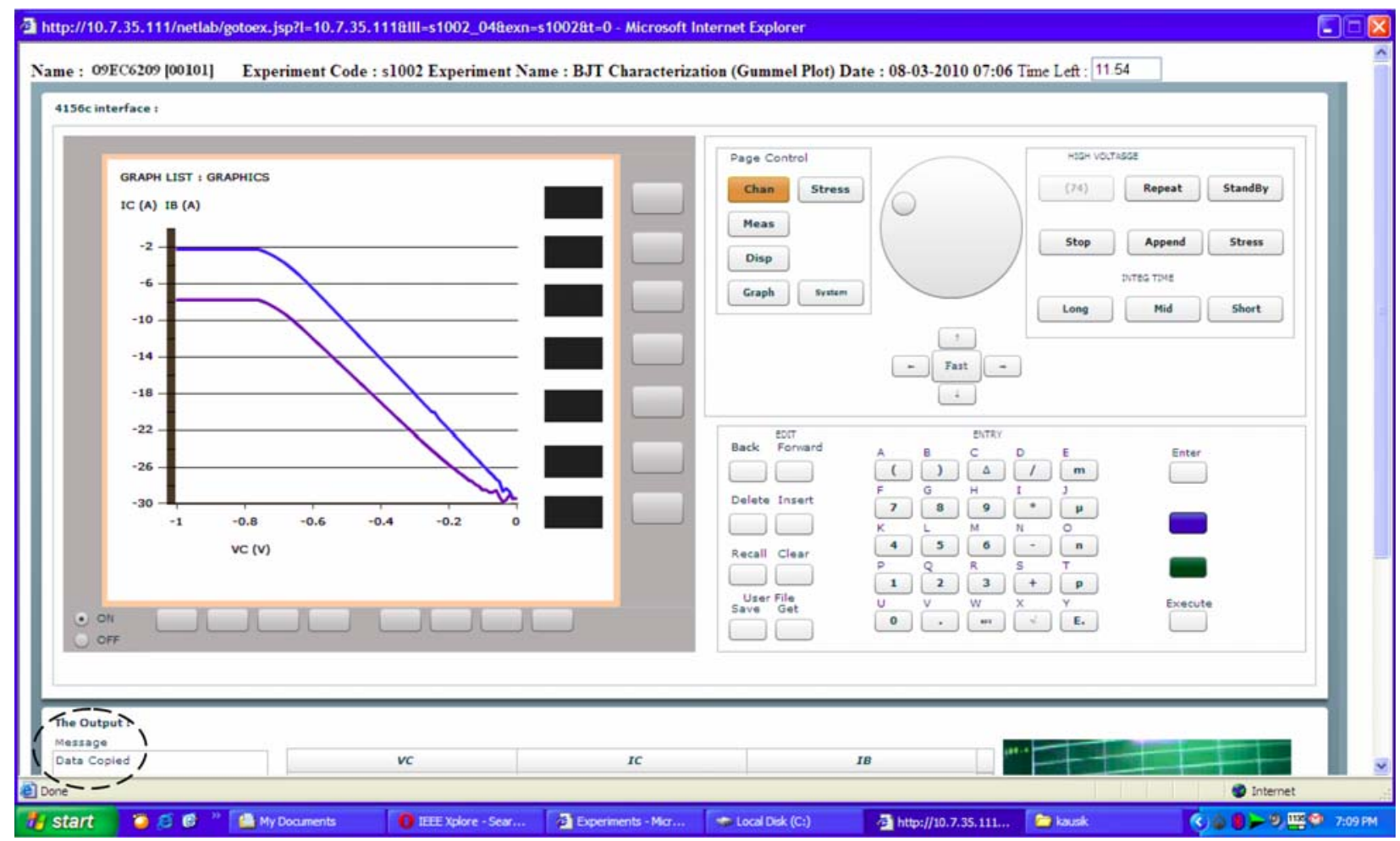

Figure 6. Measurement of BJT Forward Gummel characteristics in MVL lab. 


\section{SPECIAL FOCUS PAPER \\ DAta Handling TeChNiques IN ONLINE SEMICONDUCTOR TECHNOLOGy CAD LABORATORY}

vice under test can be extracted. The device models usually consist of a set of model equations that are either empirical or derived from device physics or a combination of both. Therefore, the design of integrated circuits is heavily dependent on circuit simulation which needs compact device models. From the measured device characteristics SPICE parameters are extracted. Several programs for parameter extraction are available on a commercial basis. In the integrated online TCAD laboratory described above, simulation program; Tool for Electronic Model Automation (TEMA) is used for the extraction of SPICE parameters [7]. SPICE parameter extraction from BJT Gummel data is shown in Figure 7.

\section{Error Handling}

This scheme has little to do about error handling. The only conditions when an error can occur are:

- the parser does not work properly if a wrong input is passed and

- no more room left in the temporary work area which is basically in the main memory.

In the first case the program will generated a garbage output or no output. Hence it has to be reported back to the user. In the second case the request is put on hold for some time before being dropped. If it is dropped then the event is notified to the user. If the request is processed, after some specified delay the output is sent back.

\section{E. DOS (Disk Operating System) based programs}

Some of the programs like SUPREM II can run only in DOS environment along with DBOS etc. Such programs are dealt by using DOS TCP/IP protocol suite for Micro- soft DOS. The TCP/IP suite gives network capabilities to the system. Of course these facilities are limited but for our purpose we use the directory sharing feature in which one folder in the Windows OS mapped to the MSDOS as a drive. A batch file run in infinite loop that keep checking for any new file in the drive i.e. folder. Whenever a request is made all relevant input files are copied to this folder. The batch file detects it and runs the program with those inputs and generates or copies the output to the drive i.e. folder. The main scheduler then reads parses and forwards the output to the user client.

\section{F. Silvaco based web enviornment}

Since the early 1980s commercial vendors such as SILVACO, ISE-TCAD, TMA and AVANTi developed their own graphical user interfaces (GUI) around existing frameworks, which facilitated the integration of process and device simulation tools by non-specialists within a wider engineering environment. The concept of general purpose process and device simulators that allows flexible simulation of different structures in different technologies became a reality. Silvaco TCAD products such as ATHENA for process simulation and ATLAS for device simulation are widely used to develop and optimize the semiconductor processes. Both ATHENA and ATLAS are physically based simulators offering predictive capability and insight into the viability of a device or process. To get SILVACO started, one needs to invoke the DECKBUILD application. DECKBUILD allows one to specify the desired process steps. Silvaco runs by operating and managing several executable files. The management is done by a GUIAppstarter program.

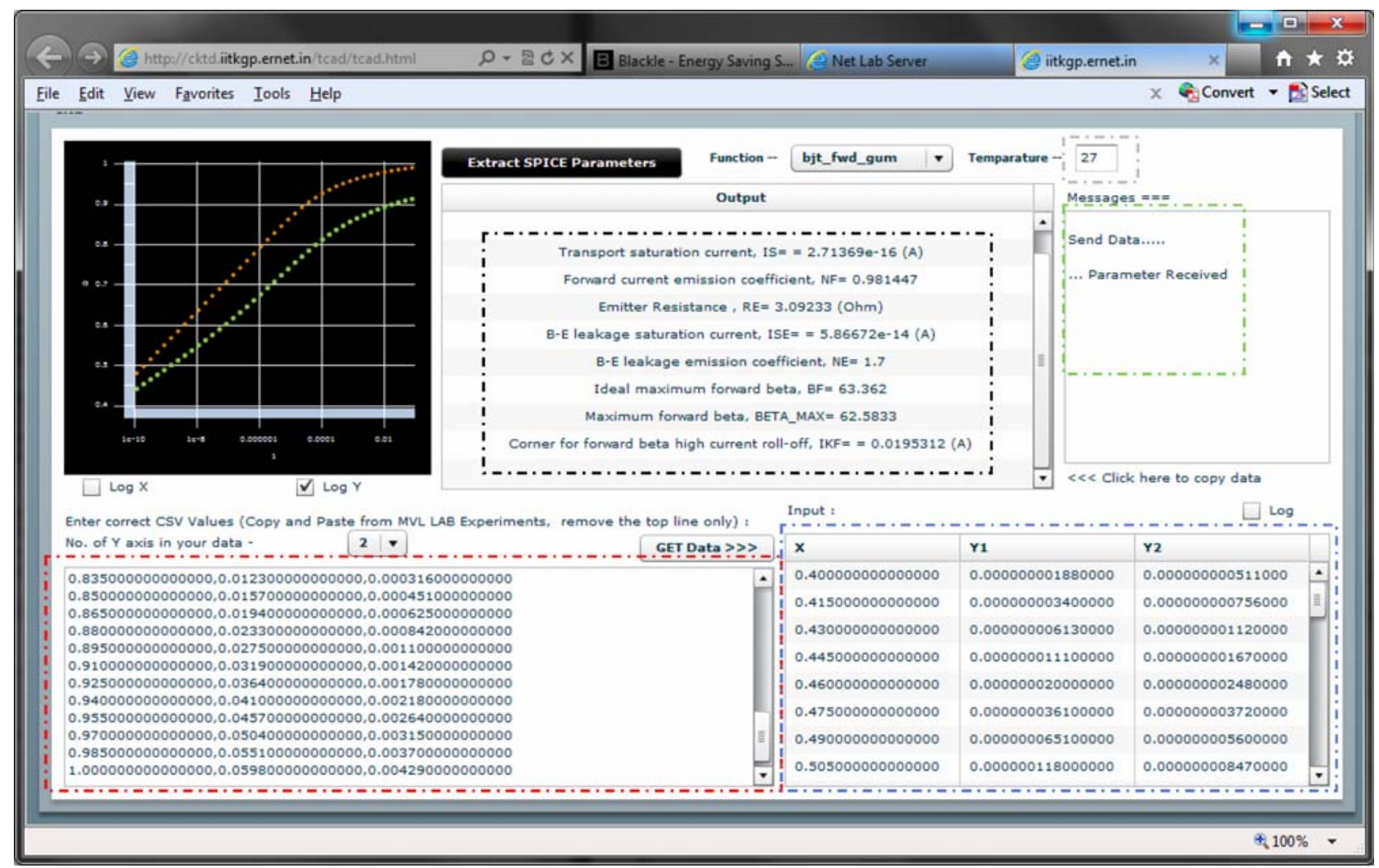

Figure 7. SPICE parameter extraction from Gummel plot. (a) Red enclosure - input data. (b) Blue enclosure - parsed input. (c) Green enclosure Messages about the request. (d) Black Enclosure - Output. (e) Grey enclosure - additional parameters along with the inputs data. 


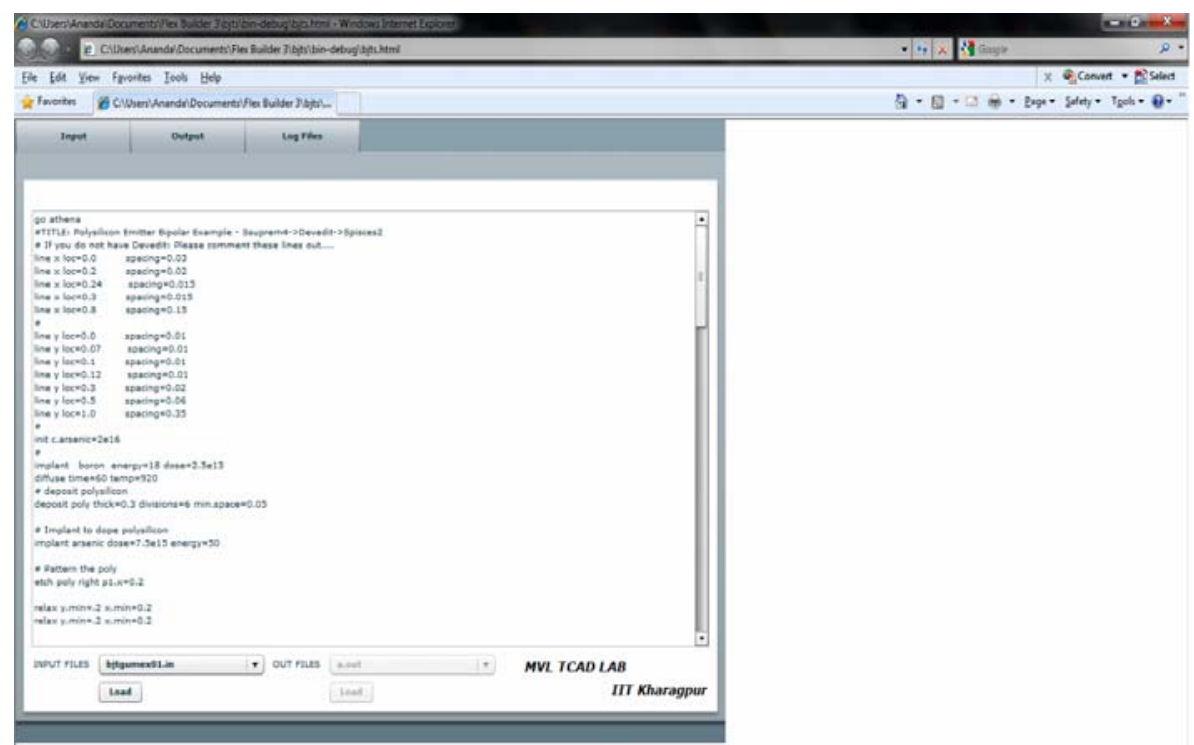

Figure 8. Input file for Silvaco web interface.

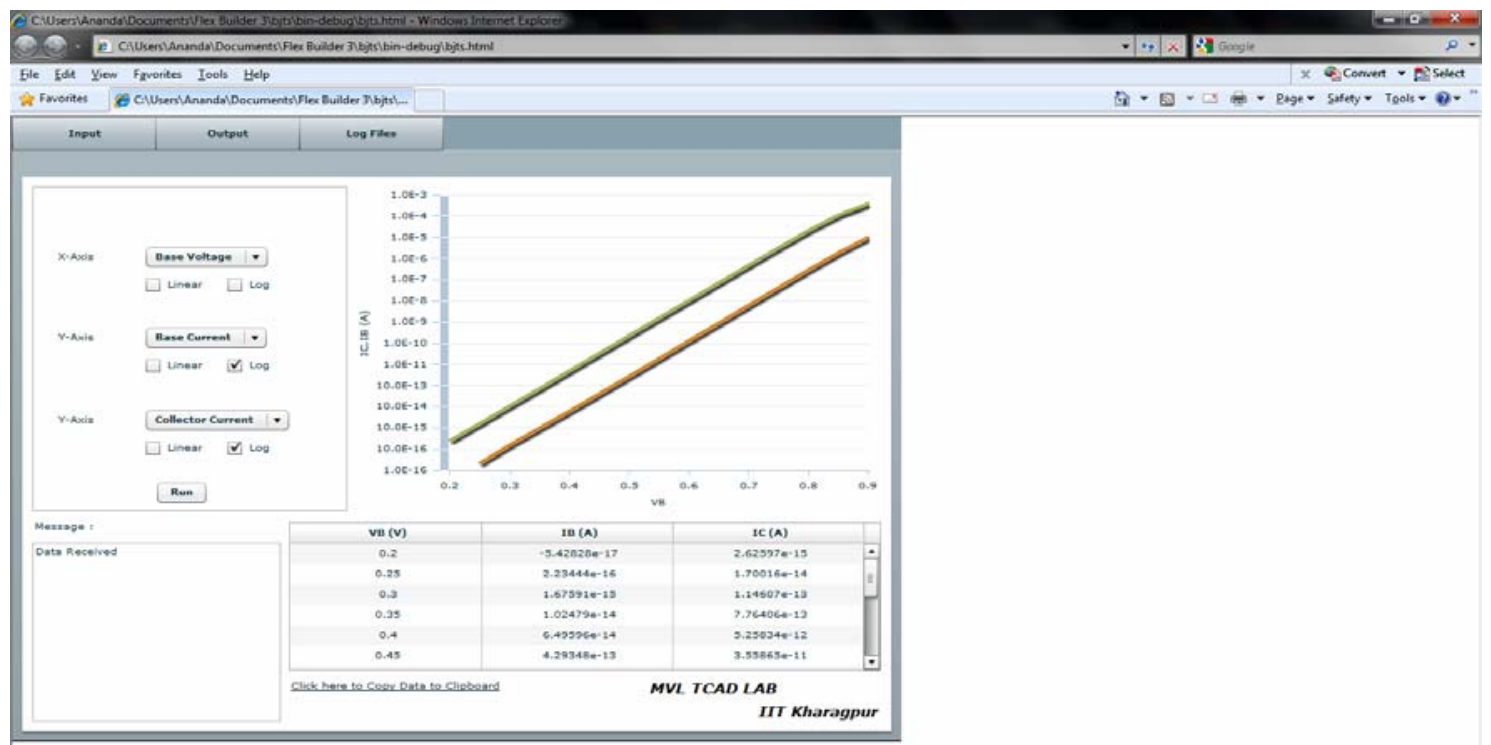

Figure 9. The output (LOG) files from Silvaco web interface.

However, individual executable file can also be operated on their own as they can read input file and produces output in text format. Hence using the method described in the earlier Section, we can deploy the Silvaco programs on the web. The Silvaco programs are mainly centered on the deckbuild.exe which takes command line input as:

deckbuild.exe -run <input filename> [-noplot] [-outfile <filename> ]

The input file is essentially a general Silvaco input file. '-noplot' indicates that no plot i.e. Tonyplot programs will not be called even if the code contains such command. The deckbuild.exe can be called from inside any java program and the output files can read and parsed.

However, Silvaco uses its own format of saving LOG files and STR files. Hence the output commands in Silvaco input file code are always kept static such that the output remains in the same format always. The value or parameters may be changed resulting in change of output file values, however, keeping the overall structure of the file same. We can parse the LOG file and output files to find necessary data and send them back to the user (see Figures 8 and 9).

Figure 8 shows the ATLAS input file for the generation of Gummel characteristics of a NPN transistor. Figure 9 shows the Gummel plot. However, Silvaco can run only one instance of simulation at a time and thus the usage of the system has to be scheduled such the no two users can access the system at a time. Also, Silvaco is a proprietary product hence its usage cannot be permitted beyond the license limits.

\section{CONCLUSIONS}

Virtual Wafer Fabrication (VWF) has become an integral part of semiconductor industry. The possibility of teaching semiconductor manufacturing in university environment in a highly cost-effective manner by taking the advantages of high speed internet and available TCAD tools has been explored. The integrated TCAD laboratory will be a support for the integrated circuit design environments, since the student can switch between the meas- 
urement and simulation environments. A measurementbased Microelectronics and VLSI Engineering Laboratory integrated with simulation based Technology CAD laboratory is described. Selection criteria for a suitable database for visualization and analyses of the generated data from process/device simulations are reported. Microelectronic courses can now be taught efficiently and cost-effectively by using process and device simulation tools without any physical process laboratory setup. With an application like this one can build a framework of open source or low cost TCAD tools from past and present for TCAD education.

\section{REFERENCES}

[1] Maiti, A.: NETLab: An Online Laboratory Management System. iJOE, vol. 6, 31-36, (2010).

[2] Seinauskas, R.: A Distance Laboratory for Computer-Aided Design, Proc. Microelectronic Systems Education, 1997,pp. 107-108, 1997. http://dx.doi.org/10.1109/MSE.1997.612569

[3] Fasching, E., Halama, S. and Selberherr, S.: (editors) Technology CAD Systems. Vienna: Springer-Verlag (1993).

[4] Dutton, R. and Yu, Z.: Technology CAD - Computer Simulation of IC Processes and Devices. Boston: Kluwer Academic Publishers (1993).
[5] Maiti, C. K., and Armstrong, G. A.: TCAD for Si, SiGe and GaAs Integrated Circuits. London: IET Press (2008).

[6] Travis, J.: Internet Applications in LabVIEW. Upper Saddle River, NJ: Prentice-Hall (2000).

[7] TEMA, (2010). Meridian Software Technology (P) Ltd. User manual.

\section{AUTHORS}

Ananda Maiti is with the Vellore Institute of Technology, School of Computing Science, Vellore 632014 TN India (e-mail: anandamaiti@live.com).

B. K. Tripathy is a senior professor with the Vellore Institute of Technology, School of Computing Science, Vellore 632014 TN India (e-mail: tripathybk@vit.ac.in).

The Technology CAD laboratory is available at: http://tcada.iitkgp. ernet.in/netlab/.

This article is an extended version of a paper presented at the International Conference on Remote Engineering \& Virtual Instrumentation (REV2011), held at Transylvania University, Brasov, Romania, June 28 July 1, 2011. Received 19 July 2011. Published as resubmitted by the authors 28 September 2011. 